\title{
Determination of drag coefficient for TOYOTA car model (Using Strain Gauge Method)
}

\author{
Abdessamed Kacem, Ali Najim Abdullah \\ ${ }^{I}$ Ministry Of Higher Education/ Baghdad University \\ ${ }^{2}$ Ministry Of Higher Education/ Al-Rafidain University College
}

\begin{abstract}
The flow field around an automobile is very complex, characterized by a high degree of three dimensionality, flow separation, reattachment and vortex formation. Flow visualization as well as flow simulation are helpful tools during the aerodynamic design of vehicles. The research-undertaken deals with an experimental estimation of $C_{D}$ (drag coefficient) for TOYOTA car,(scale 1/20) using the strain gauge method ( FLA-6-11 type, 120Q, 2.12 gauge factor, half-bridge connection), which was proved to be practical and reasonably accurate. Experiments were run within a subsonic aspiration wind tunnel, covering an air speed up to $33 \mathrm{~m} / \mathrm{s}$ (i.e., Reynolds number $5.7 \times 10^{5}$ ). Results for drag coefficient were obtained in the range of 1.10 to 0.53 . It was noted that the magnitude of $C_{D}$ decreased from 1.10 at $21.18 \mathrm{~m} / \mathrm{s}$ to 0.53 at $33.00 \mathrm{~m} / \mathrm{s}$ (i.e., decrease of drag coefficient by about 50\%). Comparison of our results with those given by other authors is satisfactory.
\end{abstract}

Key words: Strain gauge, Drag coefficient, Wind tunnel, Aerodynamics of automobile, Static loading, Dynamic loading, Half bridge connection.

\section{Introduction}

Recently the incentive to reduce the aerodynamic drag of road vehicles has increased again. Different methods to estimate drag coefficient of cars have been utilized in the past ${ }^{[1,2]}$. In the present paper, we use strain gauges with bending moment diagram in order to estimate drag coefficient, the matter that proved to be practical, accurate, and easy.

It is known that loads acting transversely to the plane of a large dimension cause a member to bend. A bar member subjected to this loading is called a beam. In order to resist these loads, a beam must be supported at one or positions along its length. If a beam has one end built-in, it is called a cantilever ${ }^{[3]}$. We used this idea in order to fix a model of a vehicle at a free end of a cantilever, and estimate the drag coefficient from bending moment diagram of the beam.

\section{The Experimental Equipment and Instrumentation}

A subsonic wind tunnel, aspiration type, with a maximum speed of $33 \mathrm{~m} / \mathrm{s}$, was used. Its cross section and active length are respectively: $230 \times 230 \mathrm{~mm}^{2}$ and $500 \mathrm{~mm}^{[4]}$. Four strain gauges, FLA-6-11, $120 \Omega, 2.12$ $\pm 1 \%$ gauge factor, wire gauge type were used, with adhesive P-2, and coefficient of thermal expansion $=11.8 \times 10^{-6} /{ }^{\circ} \mathrm{C}$. The temperature coefficient of gauge factor is $+0.1 \pm 0.05 \% / 10^{\circ} \mathrm{C}^{[5]}$. The sting made of hot rolled, medium carbon steel $(0.45 \% \mathrm{C})$, damped effect, $\mathrm{E}=203.4 \times 10^{9} \mathrm{~N} / \mathrm{m}^{2}$, and $\mathrm{I}_{\mathrm{z}}=1.4426 \times 10^{-10} \mathrm{~m}^{4}$.

A model reproducing a TOYOTA, made from PVC, scale $1 / 20$, and blockage ratio of $16 \%$ was tested. The extensometer bridge which was used, was provided with internal impedance of $120 \Omega$ to $500 \Omega$, the range of \pm 20000 points, maximum resolution: $1 \mu \Omega / \Omega$, and Amplificatory linearity is $0.002 \%$. The gauge factor regulator is 1 to 5 for 4 digits, and the excitation stability is $0.01 \%$. The branching type is a half-bridge and full-bridge with analogical exit of $0-2 \mathrm{~V}$ for $0-20000 \mu \Omega / \Omega$. The minimum charge is $2000 \Omega$, and passer band of analogical exit is 0 to $10 \mathrm{KHz}^{[6]}$.

\section{The Experimental Procedure}

Dimensions of the sting were chosen so as to reproduce minimum strain that we can read it via strain gauges. The model was fixed at the reference point of the sting as shown schematically in (fig.1a).

From the bending moment diagram, (fig. $1 \mathrm{~b})$, we can write $\mathrm{M}_{\mathrm{o}}=\mathrm{F}_{\mathrm{Y}} \cdot \mathrm{X}_{\mathrm{C}}+\mathrm{F}_{\mathrm{X}} \cdot \mathrm{Y}_{\mathrm{C}}$. The value of $\left(\mathrm{F}_{\mathrm{Y}} \cdot \mathrm{X}_{\mathrm{C}}\right)$ approaches to zero ${ }^{[3]}$. We need two equations in order to find the two unknowns $F_{X}$ and $M_{O}$, and these two equations could be obtained via the sting in its vertical position. Experimental procedure for TOYOTA model is shown in fig. (2).

\section{1-3-1 Drag force and fluid velocity calculation}

The model and four strain gauges were fixed-as shown before in (fig.1a)-at reference point $(\mathrm{O})$, (B), and (A) respectively. $L_{a}$ is the distance of strain gauge (A) from the reference point $(O) . L_{b}$ is the distance of strain gauge (B) from the reference point $(\mathrm{O})$. From the bending moment diagram, (fig.1b), we have: 


$$
\begin{aligned}
& M_{A}=M_{O}+F_{X} \cdot L_{a} \\
& M_{B}=M_{O}+F_{X} \cdot L_{b}
\end{aligned}
$$

$\mathrm{M}_{\mathrm{A}}, \mathrm{M}_{\mathrm{B}}$ have direct relationship with readings of strain gauges $\varepsilon_{\mathrm{A}}$, $\varepsilon_{\mathrm{B}}$ respectively as shown in subsequent equations:

$$
\begin{aligned}
& \varepsilon_{\mathrm{A}}=\sigma_{\mathrm{A}} / \mathrm{E}=\mathrm{M}_{\mathrm{A}} \cdot \mathrm{h} / 2 \mathrm{I}_{\mathrm{z}} \cdot \mathrm{E} \quad \longrightarrow \quad \mathrm{M}_{\mathrm{A}}=2 \mathrm{I}_{\mathrm{z}} \mathrm{E} \varepsilon_{\mathrm{A}} / \mathrm{h} \\
& \varepsilon_{\mathrm{B}}=\sigma_{\mathrm{B}} / \mathrm{E}=\mathrm{M}_{\mathrm{B}} \cdot \mathrm{h} / 2 \mathrm{I}_{\mathrm{z}} \cdot \mathrm{E} \quad \Longrightarrow \quad \mathrm{M}_{\mathrm{B}}=2 \mathrm{I}_{\mathrm{z}} \mathrm{E} \varepsilon_{\mathrm{B}} / \mathrm{h}
\end{aligned}
$$

Where $I_{z}$ represents the second moment of area for the sting around Z-axis as shown schematically in (fig.3). By solving equations (1) and (2), we can find the unknowns $\mathrm{F}_{\mathrm{X}}$ and $\mathrm{M}_{\mathrm{O}}$. Drag coefficient could be estimated by the relationship:

$$
\mathrm{C}_{\mathrm{D}}=\mathrm{F}_{\mathrm{X}} / 0.5 \rho \mathrm{V}_{\infty}^{2} \mathrm{~A}
$$

And fluid velocity (air-speed) could be estimated by:

$$
V_{\infty}=\sqrt{2 g\left(\rho_{w} / \rho_{a}\right) \Delta h}
$$

\section{1-3-2 Deviation analysis for measurements}

From equation (6):

$$
\begin{aligned}
& \mathrm{V}=\text { constant } \mathrm{x} \\
& \ln \mathrm{V}=\ln \text { constant }+\ln \mathrm{H}^{1 / 2}
\end{aligned}
$$

By differentiating the equation logarithmically

$$
\mathrm{dV} / \mathrm{V}=0.5 \times \mathrm{dH} / \mathrm{H}
$$

Where $\mathrm{dH}$ represents the absolute error ratio in total pressure head that could be estimated:

$$
\mathrm{dH}=\sqrt{\left(H-H_{m}\right)^{2}+(\delta H)^{2}}
$$

Where $\mathrm{H}$ represents the total head.

Deviation analysis for drag force measurements could be estimated from:

$$
\mathrm{dF}_{\mathrm{X}} / \mathrm{F}_{\mathrm{X}}= \pm \sqrt{\left(d \varepsilon_{A} / \varepsilon_{A}\right)^{2}+\left(d \varepsilon_{B} / \varepsilon_{B}\right)^{2}}
$$

Deviation analysis for drag coefficient could be estimated from:

$$
\mathrm{dC}_{\mathrm{D}} / \mathrm{C}_{\mathrm{D}}= \pm \sqrt{\left(d F_{X} / F_{X}\right)^{2}+(2 d V / V)^{2}}
$$

\section{Results and discussion}

Figure(4) shows the results of the wind tunnel calibration. Good stability in velocity distribution within the working section, was obtained.

Experiments were run at an air speed from $21.17 \mathrm{~m} / \mathrm{s}$ to $33.00 \mathrm{~m} / \mathrm{s}$ (i.e., Reynolds number from 3.67 $10^{5}$ to $5.7210^{5}$ ), results for drag coefficient were obtained in the range of 1.10 to 0.53 as shown in figure (5). It was noted that the value of CD decreased from 1.10 at $21.17 \mathrm{~m} / \mathrm{s}$ to 0.53 at $33.00 \mathrm{~m} / \mathrm{s}$ (i.e., decrease of drag coefficient by about $50 \%$ within the range of an air speed of $12 \mathrm{~m} / \mathrm{s}$ ).

Figure (6) shows that $\mathrm{dV} / \mathrm{V}$ ratio varies from $\pm 3.57 \%$ to $\pm 1.47 \%$ (i.e., decreases by $59 \%$ ), while $\mathrm{dF}_{\mathrm{X}} / \mathrm{F}_{\mathrm{X}}$ varies from $\pm 3.66 \%$ to $\pm 1.42 \%$ (i.e., decreases by $61 \%$ ), and $\mathrm{dC}_{\mathrm{D}} / \mathrm{C}_{\mathrm{D}}$ ratio varies from $\pm 8.02 \%$ to \pm $3.26 \%$ (i.e., decreases by $59 \%$ ), at the working range of an air speed.

For the undertaken model, the separation tends to occur when air flows from a low pressure to a high one, which is known as an adverse pressure gradient. Conversely, a flow from a high pressure to a low one, is known as a favorable pressure gradient, which is not only inhibits the separation but also slows down the rate of boundary layer growth and delays the transition. A flow separation is particularly and likely to occur when the air tries to go around a very sharp bend, Figure $(7 \& 8)$.

Figure (9) shows the flow visualization around TOYOTA model with re-circulating bubble or spiral vortices at the rear. Figure (10) shows the scheme for the trailing vortices at the rear part of the vehicle.

\section{Conclusion}

The re-circulating bubble or spiral vortices determine the drag and stability. From the flow visualization that consolidated with the strain gauge method results, it can be seen that as the size of vortices are small, the drag coefficients are low.

\section{Notations}

$\mathrm{F}_{\mathrm{X}}$ : Total drag force $(\mathrm{N})$.

$\mathrm{F}_{\mathrm{Y}}$ : Lift force $(\mathrm{N})$.

$\mathrm{M}_{\mathrm{A}}$ : moment at A (N.m).

$\mathrm{M}_{\mathrm{B}}$ : moment at B (N.m). 
$\mathrm{M}_{\mathrm{O}}$ : Pitching moment (N.m).

$\varepsilon_{\mathrm{A}}$ : Strain at strain gauge $\mathrm{A}$ ( $\mu$ strain).

$\varepsilon_{B}$ : Strain at strain gauge B ( $\mu$ strain).

E: Young modulus of elasticity $\left(\mathrm{N} / \mathrm{m}^{2}\right)$.

$\left(\mathrm{X}_{\mathrm{C}}, \mathrm{Y}_{\mathrm{C}}\right)$ :Centroid co-ordinates of the sting.

$\mathrm{I}_{\mathrm{Z}}$ : Second moment of area $\left(\mathrm{m}^{4}\right)$.

$\sigma_{\mathrm{A}}$ : Stress at $\mathrm{A}\left(\mathrm{N} / \mathrm{m}^{2}\right)$.

$\sigma_{\mathrm{B}}$ : Stress at B $\left(\mathrm{N} / \mathrm{m}^{2}\right)$.

$\mathrm{b}$ : Width of beam $(\mathrm{m})$.

h: Thickness of beam (m).

$\rho_{\mathrm{a}}$ : Air density $\left(\mathrm{kg} / \mathrm{m}^{3}\right)$.

$\rho_{\mathrm{w}}$ : Water density $\left(\mathrm{kg} / \mathrm{m}^{3}\right)$.

$\mathrm{V}_{\infty}$ : Undisturbed air flow $(\mathrm{m} / \mathrm{s})$.

g: Gravitational acceleration $\left(\mathrm{m} / \mathrm{s}^{2}\right)$.

$\Delta \mathrm{h}$ : Head difference $\left(\mathrm{m} \mathrm{H}_{2} \mathrm{O}\right)$.

$\mathrm{C}_{\mathrm{D}}$ : Drag coefficient.

A: The model frontal area $\left(\mathrm{m}^{2}\right)$.

$\mathrm{L}_{\mathrm{a}}$ : The distance of strain gauge A from the reference point $(\mathrm{O})$.

$\mathrm{L}_{\mathrm{b}}$ : The distance of strain gauge $\mathrm{B}$ from the reference point $(\mathrm{O})$.

Subscript (O): Reference point (model fixing position on the sting at the back of the model).

\section{References}

[1]. Itsuhei Kohri, Teppei Yamanashi \& Takayoshi Nasu, "Study on the transient behavior of the vortex structure behind Ahmed body",SAE 2014.

[2]. Muzafferuddin Mahmood,"flow visualization in Wind Tunnels", 2011.

[3]. M.J.Iremonger,"Basic Stress Analysis", 1984.

[4]. Delta $\triangle$ Lab, "Soufflerie Subsonique a aspiration EA600", France 2014

[5]. Tokyo Sokki Kenkyujo Co. "TML Strain Gauge Test Data", Japan,2015.

[6]. Delta $\triangle$ Lab, “Pont D’Extensometrie EI 616”, France 2014.

[7]. Simone Sebben, Tim walker \& Christoffer, "Fundamentals basic principles in road vehicle Aerodynamics and design", April 2014.

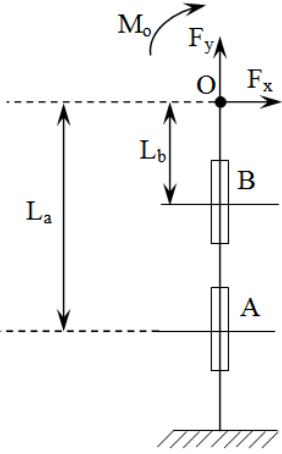

(a)

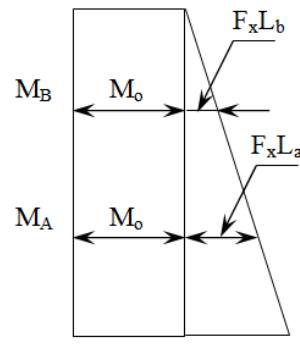

(b)

Figure (1): (a) The vertical position of the sting (schematically)

(b) The bending moment diagram for the sting

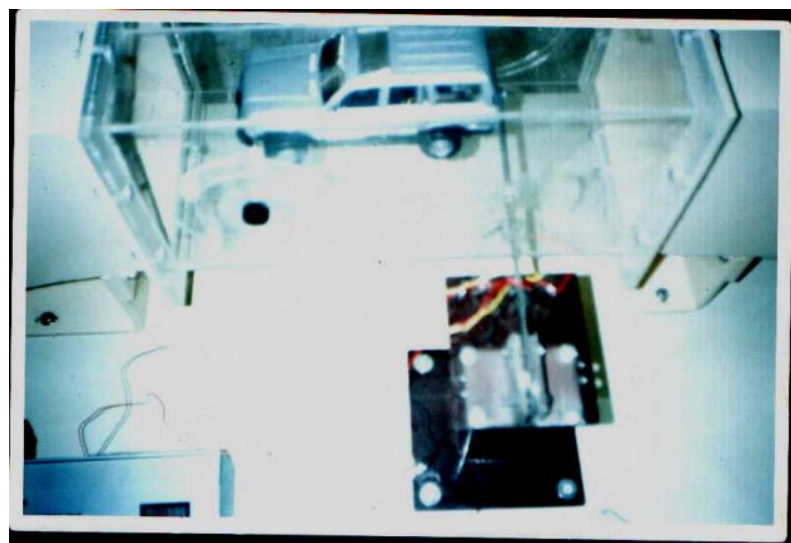

Figure (2): Experimental procedure for TOYOTA model 


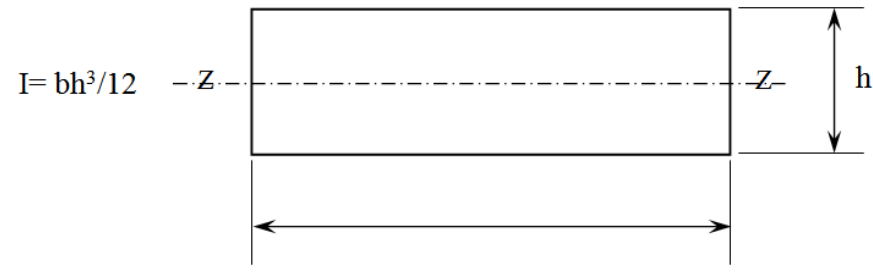

Figure (3): The second moment of area for the sting

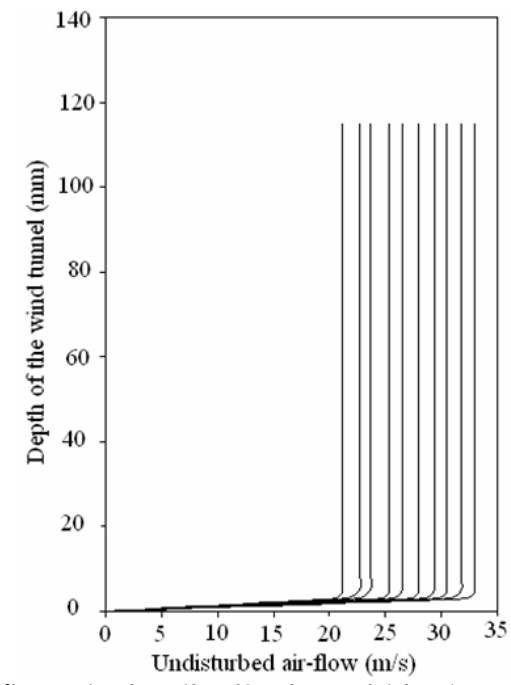

Figure (4): Velocity distribution within the test section.

From calibration of the wind tunnel

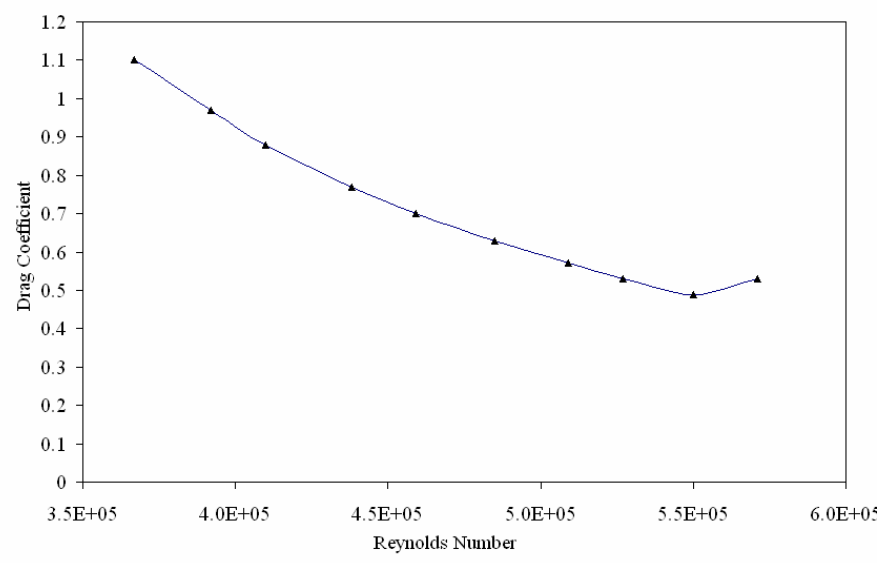

Figure (5): Drag coefficient versus air speed for TOYOTA model

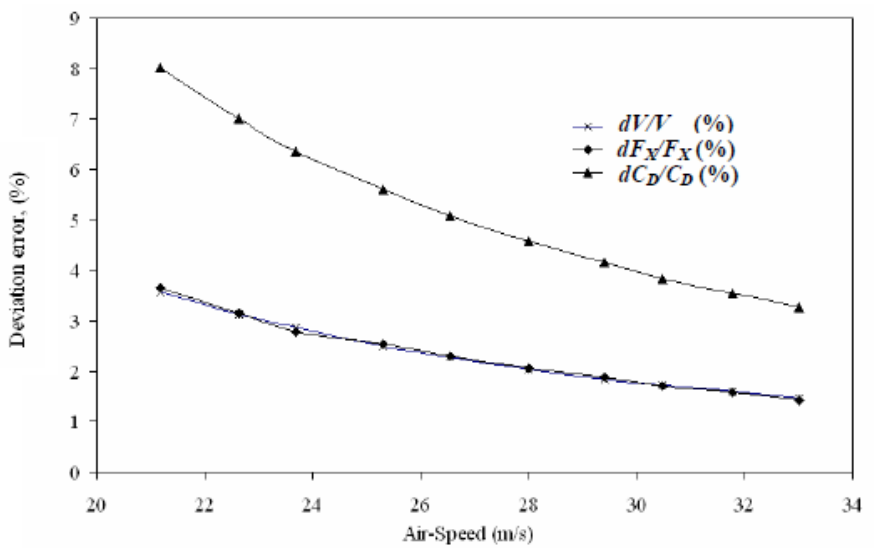

Figure (6): Variation of errors deviation with an air-speed for TOYOTA mode 

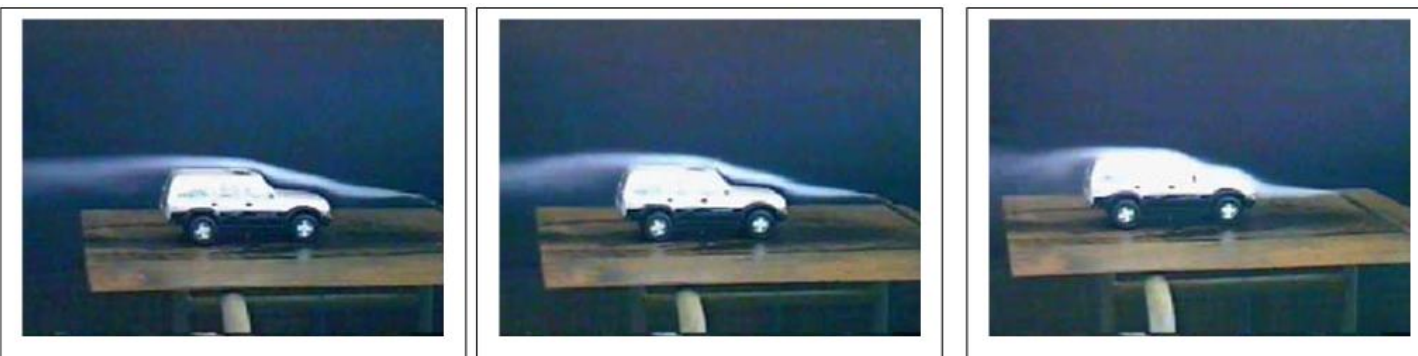

Figure (7): Flow visualization around TOYOTA model
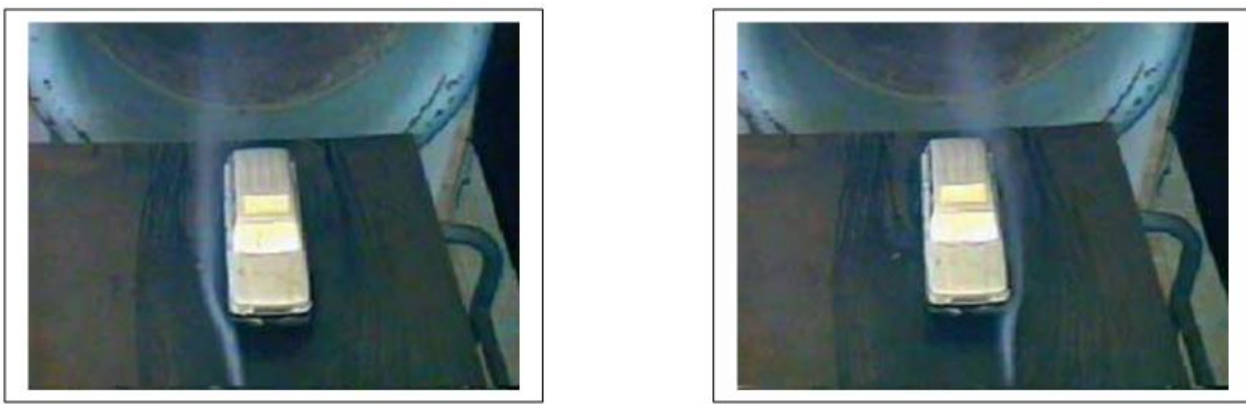

Figure (8): Attached flow at the right and left sides for TOYOTA model
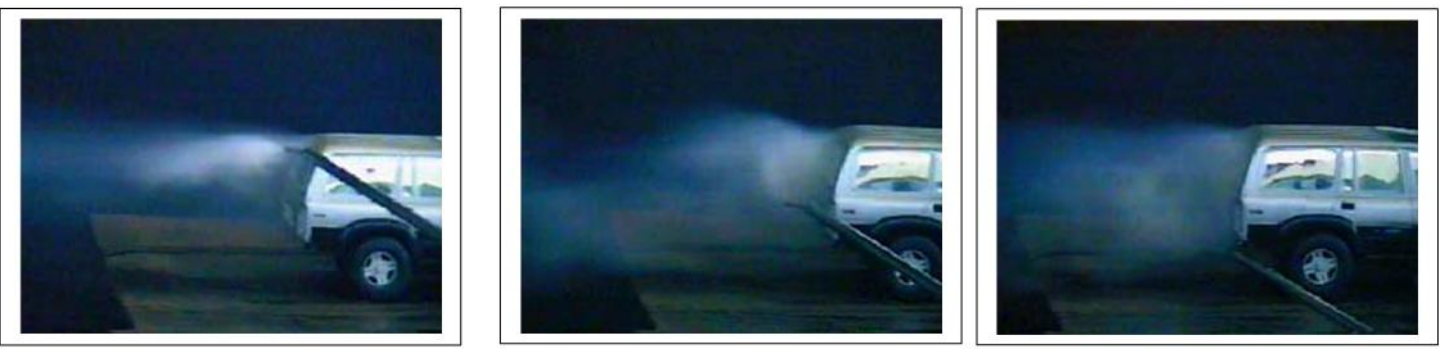

Figure (9): Re-circulating bubble or spiral vortices at the rear part of TOYOTA model

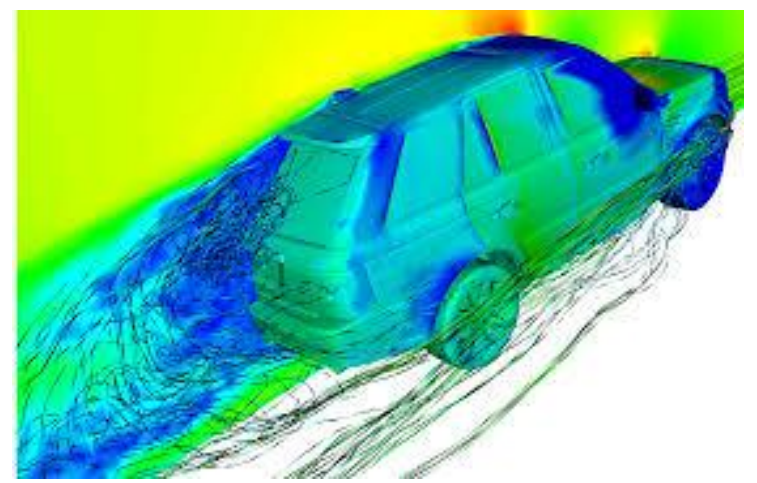

Figure (10): Scheme for the trailing vortices at the rear part of the vehicle ${ }^{[7]}$ 